\title{
Analysis of the Impact of Different Parameter Settings on Wireless Sensor Network Lifetime
}

\author{
Muhammad Usman Younus \\ Institut de Recherche en Informatique de Toulouse (IRIT) \\ Université Paul Sabatier, Toulouse, France
}

\begin{abstract}
The importance of wireless sensors is increasing day by day due to their large demand. Sensor networks are facing some issues in which battery lifetime of sensor node is critical. It depends on the nature and application of the wireless sensor network and its different parameters (sampling frequency, transmission frequency, processing power and transmission power). In this paper, we propose a new and realistic model to show the effect of energy consumption on a lifetime of wireless sensor nodes. After analyzing the model behavior, we are able to find out the sensitive parameters that are highly effective for lifetime of sensor nodes.
\end{abstract}

Keywords-Wireless sensor network; energy consumption; lifetime; WSN parameters; transmission frequency; sampling frequency

\section{INTRODUCTION}

The low cost, the low implementation complexity, and the flexibility of wireless sensor networks (WSNs) account for them who are employed in industrial, residential and commercial sectors. Since the last twenty years, there has been a widespread growth in wireless communication technology. Thus, it became a significant part of our lives. The applicability of WSNs extends from telephony and data transfer to non-sensing monitoring approaches. In spite of being a successful and vast field, there exist certain complications like battery lifetime and reliability that need to be sorted. An important parameter to measure the performance of a wireless network is its battery life [1] because wireless sensors have limited battery capacity. In some applications, after the deployment of a sensor, the battery cannot be replaced. So, the problem of high energy consumption is very critical in WSN for continuity of the network communication.

A long battery life is a plus point for wireless local area network (WLAN) cards or cell phones, while it is an obligation for large-scale sensor networks. It is more difficult to replace a battery with hundreds of sensor nodes all the time [2]. This was the main reason which attracted researchers to study a way out to devise a lifetime network for WSNs.

The energy is the main limiting factor for a battery life. The power consumption of transceivers is taken as energy with respect to time. The circuit power is vital determinant for the analysis of battery life as RF of short-range that has a carrier frequency in Giga Hertz. In addition to the aforementioned, other parameters like sensing duration, transmission duration, receiving duration, transmitting frequency, sampling frequency, channel condition, modulation, and data rate must be considered as this effect transmits power of RF, architecture and operating time of the transceiver [3].

A lot of work has already been done in the field of WSN to reduce the energy consumption of sensor nodes and enhance the lifetime with different ways which are discussed in section II. To the best of our knowledge, no one is focusing on whole WSN energy consumption parameters. In this paper, we are presenting a new and realistic model, in which WSN energy consumption parameters are discussed in detail that will help to enhance the lifetime of the wireless sensor node.

Following is a general layout of our paper: in Section II the literature review is being discussed; in Section III the mathematical formulation of energy consumption of wireless sensor nodes are described; Model analysis, its performance, and discussion are explained in Section IV, while concluding remarks are given in Section V.

\section{LITERATURE REVIEW}

This section provides the state-of-art in various fields of research regarding battery lifetime of WSNs. Many studies have been focused on the estimation and the issue of battery lifetime regarding different types of applications such as data networks, commercial sector, personal area network, free internet connection sharing, etc. Many researchers are working in this field for the last few decades. Some of the remarkable works are discussed below.

In [4], the researchers carried out a study on modeling the lifetime of wireless sensors. According to this paper, energy consumption and Remaining Lifetime of Individual Sensor (RLIS), Remaining Lifetime of whole Sensor Network (RLSN) and Lifetime of Sensor Network (LSN) were formerly designed. On considering these models, two query protocols were introduced. These two models had two approaches: Firstly, it provides the most usable model for every type of energy consumption such as sleep/active dynamics. Secondly, it provides the efficient protocols. This paper reveals that load balancing causes the extension of the lifetime for a network.

In [5], the researchers investigated the energy consumption of wireless transceivers. According to this study, battery lifetime was considered the vital parameter to evaluate the overall performance of wireless sensor networks (WSNs). Researchers found that energy cost of transceiver electronic systems complicates the situation by putting its impact on battery lifetime. This paper basically aims at the development of energy cost for both electronic circuit and communication protocols. 
Followed by the previous work, the others tried to overcome the lapses left by them. Moreover, in [6], the authors suggested a study on the trade-off between utility and lifetime in WSNs using a cross-layer optimization approach. The trade-off model depends on optimization decomposition.

Furthermore, many researchers have demonstrated the packet transmission policies for enhancing the lifetime of WSNs. In [7], they proposed battery friendly packet transmission policies for WSNs. Their work focused on maximizing battery lifetime for those networks that are affected by delayed constraints. In their study, these packets were introduced to reach their goal.

The problem of reducing the energy consumption and enhancing battery lifetime for WSNs attracted the attention of researchers in most applications where sensors are irreplaceable. So, the researchers in [8] studied Hierarchy Energy Driven Architecture (HEDA). This approach targeted overall energy consumption cost for enhancing battery life for WSNs. Many researchers had defined the lifetime of a sensor node and that of a network differently based on nature and applicability of WSN. They had illustrated lifetime being a function of energy consumption that depends on a function of transmitting distance [9]. Additionally, some other parameters like a total number of nodes and area of sensor fields within a network account for the lifetime of WSN. Different parameters like transmission radius, number of nodes in an area and coverage area of sensor network collectively have an impact on a lifetime of WSN that follows Fermat Point based forwarding technique.

Many scientists conducted a survey on battery and solar powered wireless sensor nodes. The vast applicability of sensor nodes is being hindered by problems created through a power supply and source. Therefore, energy conservation should be targeted at first place while implementing battery/solar powered sensor nodes. Previously known batteries served as a power source for nodes [10]. The motive of this paper was to compare solar and cell powered batteries. The vital parameters being studied included capacity volume, energy density, power efficiency, and low self-discharge, shorter recharge time and lower cost in terms of weight or size.

In [11], the researchers conducted a study on mobile ad hoc networks (MANETs) for reducing the power consumption through dynamic power management techniques. These MANETs were able to communicate with other networks as it consisted of free nodes to move randomly. It also enhances the battery life by efficient consumption of power through proper routing techniques/protocols and limited battery supply to each node. The main aim of the paper was to target the energy consumption through optimal path selection. Since a few decades, this field is leading to success. In [12], they had carried out a survey in which various optimization techniques for energy consumption within WSNs were considered. The main motive of the paper was to design a model for reducing battery overhead and enhancing lifetime of WSN that exploits the concept of Epidemic Model [12]. The network monitoring was carried out through a special node which makes use of the same protocol as for communication and tracks the state of nodes at its propagation. This model can be utilized on the basis of its location sensing, positions, continuous sensing and event detection etc. This goal was achieved through numerical analysis. This literature provides fruitful ways for reduction of energy consumption and enhancement of lifetime of WSNs.

Moreover, in [13], researchers conducted a study for lifetime estimation of Wireless sensor nodes via models of an embedded analytical battery. In this study, the variable behavior of electrochemical batteries in terms of voltage and charge serves as a relevant parameter for implementation of energy policies. Currently, various hardware and software are available in a market that estimates the operational condition of a battery. Hardware approaches are comparatively costly than the software ones. This study focused on software approaches based on the temperature-dependent analytical model for estimation of voltage/charge values of WSNs. Such approaches were found successful without being affecting the overall performance of the WSNs. Still, there is more deficiency in this field of WSNs that needed to be studied.

Section III is describing the mathematical formulation of energy consumption of wireless sensor nodes and our model analysis is discussed in Section IV.

\section{ENERGY CONSUMPTION FORMULATION}

The Wireless sensor consists of some units which contain sensing, processing, transceiver, and power units as shown in Fig. 1. Each unit has different energy consumption which depends on the working of each unit.

Sensing and processing units collect the data from the environment and process it. Then the transceiver unit transmits and receives the data to the next hop in the wireless network. Power unit provides the power to each unit of a sensor.

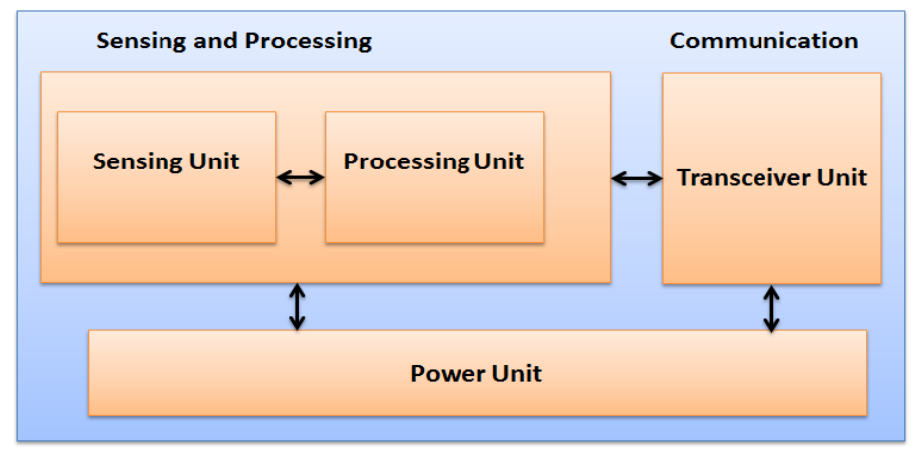

Fig. 1. Wireless sensor units.

Following is the total energy consumption of a wireless sensor node in the interval $\mathrm{t}$ :

$$
E(t)=\left\{\begin{array}{l}
E^{\text {Sens }}(t)+E^{T x}(t)+E^{R x}(t) \\
+E^{A c k T x}(t)+E^{A c k R x}(t)+E^{\text {Idle }}(t)
\end{array}\right.
$$

Equation (1) consists of the whole energy consumption sources during time $\mathrm{t}$ due to sensing and processing, data transmitting and receiving, transmitting the acknowledgment (ACK) of successful receiving data frames and during idle time energy consumption. 
Sensing and processing energy consumptions are:

$$
E^{\text {Sens }}(t)=t * f_{S} * P_{S} * \Delta t_{S} \quad \text { where, } t \leq L T
$$

According to Eqn. 2, energy consumption of a sensing unit depends on sampling frequency, processing power, sampling duration where LT is a lifetime of node which must be less or equal to the total time. Definitions of symbols are given in Table I.

Transmission energy consumption is:

$$
\begin{gathered}
E^{T x}(t)=N F_{T x}^{A v g} * P_{T x} * \Delta t_{T x} \\
N F_{T x}^{A v g}=N F_{T x} * \frac{1-\left(1-(1-\eta)^{2}\right)^{R+1}}{1-\left(1-(1-\eta)^{2}\right)}
\end{gathered}
$$

where $N F_{T x}=t * f_{T x}$ \& $t \leq L T$

Energy consumption due to transmission depends on an average number of the transmitted frame, sensor transmitting power, and transmission time duration as given in (3). Equation (4) defines the average number of frames which also include the retransmission of frames due to frame or ACK loss, where $N F_{T x}$ is the total number of frames which are transmitted during time t. All the remaining energy consumption parameter equations (i.e. Energy consumption due to reception, transmission of ACK frame, reception of ACK frame and energy consumption during idle time) will follow the average number of frames because of frames or ACK loss issues.

Energy consumption due to reception is:

$$
\begin{aligned}
& E^{R x}(t)=N F_{R x}^{A v g} * P_{R x} * \Delta t_{R x} \\
& N F_{R x}^{A v g}=N F_{T x} *(1-\eta) * \frac{1-\left(1-(1-\eta)^{2}\right)^{R+1}}{1-\left(1-(1-\eta)^{2}\right)}
\end{aligned}
$$

Energy consumption due to receiving the data depends on average number of frames, receiving power and frames receiving duration as given in (5), where (6) defines the average number of receiving frames in which $R$ times reception is included due to frame loss.

Energy consumption due to transmission of ACK frame is:

$$
E^{A c k T x}(t)=N F_{R x}^{A v g} * P_{A c k T x} * \Delta t_{A c k T x}
$$

ACK frame is used to give the confirmation message about successfully receiving frames. Due to ACK transmission, energy is consumed which depends on an average number of frame reception, its transmitting power, and ACK transmission duration as given in (7).

Energy consumption due to reception of ACK frame is:

$$
\begin{aligned}
& E^{A c k R x}(t)=N F_{A c k R x}^{A v g} * P_{A c k R x} * \Delta t_{A c k R x} \\
& N F_{A c k R x}^{A v g}=N F_{T x} *(1-\eta)^{2} * \frac{1-\left((1-\eta)^{2}\right)^{R+1}}{1-(1-\eta)^{2}}
\end{aligned}
$$

If the destination node receives frames successfully then that destination node transmits the ACK frame to give confirmation about successfully receiving frames. Equation (8) defines the energy consumption due to ACK frame reception, which includes the average number of ACK frames, power consumption due to reception of ACK frames and receiving of ACK frames duration. An average number of ACK frames also include $\mathrm{R}$ retransmissions which are due to loss of ACK or data frames loss during communication as given in Equation (9).

\section{TABLE I. NOTATION DEFINITIONS}

\begin{tabular}{|l|l|}
\hline Symbol & Definition \\
\hline$f_{s}$ & Sampling frequency \\
\hline$P_{S}$ & Power consumption due to samples processing \\
\hline$\Delta t_{S}$ & Sampling duration \\
\hline$L T$ & Lifetime \\
\hline$N F_{T x}^{A v g}$ & Average number of transmitted frames \\
\hline$f_{T x}$ & Transmission frequency \\
\hline$P_{T x}$ & Power consumption due to transmission \\
\hline$\Delta t_{T x}$ & Duration of transmission \\
\hline$N F_{R x}^{A v g}$ & Average number of reception frames \\
\hline$P_{R x}$ & Power consumption due to reception \\
\hline$\Delta t_{R x}$ & Duration of reception \\
\hline$N F_{A c k R x}^{A v g}$ & Average number of ACK reception \\
\hline$P_{A c k T x}$ & Power consumption due to ACK transmission \\
\hline$\Delta t_{A c k T x}$ & Duration of ACK transmission \\
\hline$P_{A c k T x}$ & Power consumption due to ACK reception \\
\hline$\Delta t_{A c k T x}$ & Duration of ACK reception \\
\hline$P_{I d l e}$ & Power consumption during Idle \\
\hline$\eta$ & Probability of error \\
\hline$R$ & Number of retransmission \\
\hline$V_{s}$ & Sensor supply voltage \\
\hline & \\
\hline & \\
\hline
\end{tabular}

Energy consumption during the idle situation is:

$$
E^{\text {Idle }}(t)=\left\{\begin{array}{l}
P_{\text {Idle }} *\left(t-\left(t^{*} f_{S} * \Delta t_{S}\right)-\left(N F_{T x}^{A v g} * \Delta t_{T x}\right)-\right. \\
\left(N F_{R x}^{A v g} * \Delta t_{R x}\right)-\left(N F_{R x}^{A v g} * \Delta t_{A c k T x}\right)- \\
\left.\left(N F_{A c k R x}^{A v g} * \Delta t_{A c k R x}\right)\right)
\end{array}\right.
$$

Very low energy is consumed when no process is carried out during this situation that's calculated by (10). Energy consumption during idle situation depends on power consumption rating of a node and idle duration.

However, the lifetime of sensor node is defined by: 
Life time of sensor node $=\frac{\text { Initial battery energy }}{\text { Total power consumed }}$

Here we have considered that average battery voltage rating is known. The unit of an initial battery is Joule and power is in Watt. Here in next section, we are going to analyze the performance of the model in term of a lifetime.

\section{PERFORMANCE ANALYSIS OF MODEL AND DiSCUSSION}

In this section, we will see how different parameters affect the battery lifetime of sensor node. Our results are based on analysis and corroborated by MATLAB Work. We followed the Tmote Sky sensor device rating [14], [15] which is Ultra low power IEEE 802.15.4 complaint wireless sensor module. This module is widely used in sensor networks for monitoring, measuring the temperature and humidity. It has high data rate (250kbps) and an onboard microcontroller with 10k RAM, $48 \mathrm{k}$ flash, an antenna with $125 \mathrm{~m}$ range outdoor and $50 \mathrm{~m}$ indoor. Due to onboard features, it is cheaper and smaller in size as compared to other motes. The parameter values are given in Table II. Our results are based on the parameters of a real device; it is expected to reflect the actual behavior of a system in terms of lifetime.

TABLE II. PARAMETERS VALUES

\begin{tabular}{|l|ll|}
\hline Symbol & Value & \\
\hline$P_{S}$ & $24 \mathrm{~mW}$ & {$[17]$} \\
\hline$P_{t x}$ & $58.5 \mathrm{~mW}$ & {$[14],[15]$} \\
\hline$P_{r x}$ & $65.4 \mathrm{~mW}$ & {$[14],[15]$} \\
\hline$P_{I d}$ & $163 \mu W$ & {$[14],[15]$} \\
\hline$V_{s}$ & $3 \mathrm{~V}$ & {$[15]$} \\
\hline$\Delta t_{S}$ & $5 \mathrm{~ms}$ & \\
\hline$\Delta t_{T x}$ & $4.2 \mathrm{~ms}$ & {$[16]$} \\
\hline$\Delta t_{R x}$ & $4.2 \mathrm{~ms}$ & {$[16]$} \\
\hline$\Delta t_{A c k T x}$ & $0.35 \mathrm{~ms}$ & {$[16]$} \\
\hline$\Delta t_{A c k R x}$ & $0.35 \mathrm{~ms}$ & {$[16]$} \\
\hline
\end{tabular}

In our model parameters, sampling frequency $f_{s}$ depends on an application because each application required a different number of samples. We are also assuming the sampling time duration.

We compared four different parameters for observation which are transmitting frequency, sampling frequency, transmission, and processing power.

Fig. 2 shows the effect of a sampling frequency $(F s)$, transmission frequency $(F t x)$, processing power $(P s)$ and transmission power $(P t x)$ on a lifetime of a sensor node. In Fig. 2(a), the effect on sampling frequency and transmission frequency at the lifetime of a sensor node is shown. The lifetime of sensor node is decreasing with increase in both sampling and transmission frequency. From this graph, we can see that transmission frequency is more effective on lifetime as compared to the sampling frequency. Lifetime is decreasing exponentially with increase in transmission frequency. However, the lifetime is also decreasing when the sampling frequency increases but not exponentially. With Ftx varying, remaining lifetime of sensor node is lower as compared to $F s$ varying scenario.

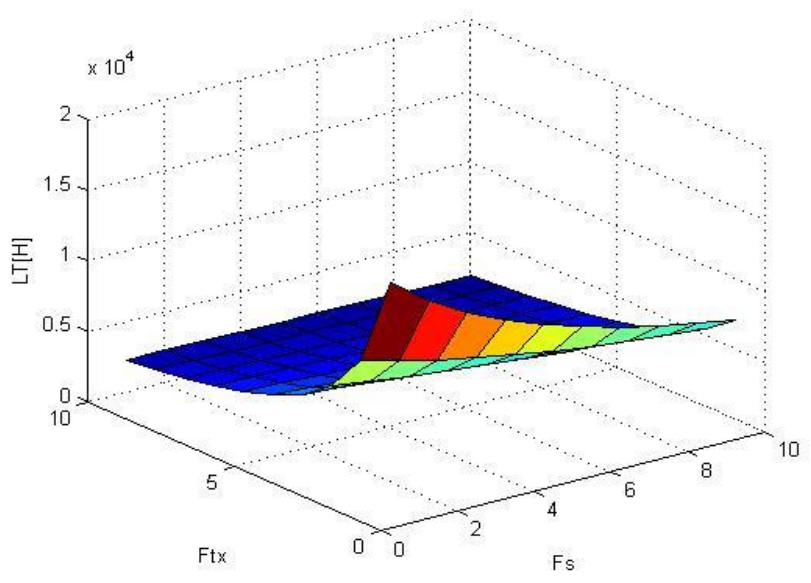

(a)

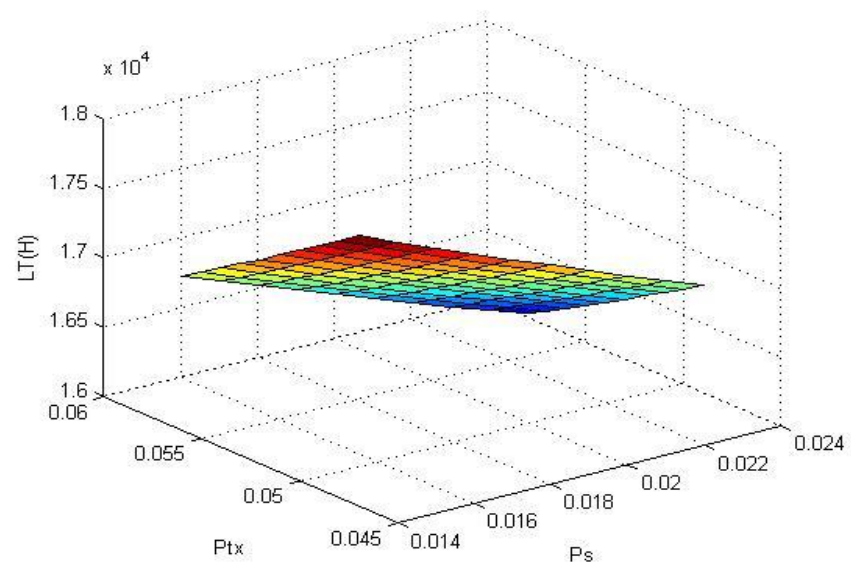

(b)

Fig. 2. Effect of frequencies and powers on node lifetime (a) Effect of sampling and transmission frequency on lifetime (b) Effect of processing and transmission power on lifetime.

In Fig. 2(b), the effect on the lifetime of a sensor node due to processing and transmission power is shown. The lifetime of sensor node is decreasing exponentially while transmission power increases. However, the lifetime of a sensor node is also decreasing when the sampling frequency increases but not exponentially. In Fig. 2(b) scenario, with Ptx varying, remaining lifetime of sensor node is lower as compared to $P s$ varying scenario.

From both Fig. 2(a) and 2(b), it can be predicted that more effective parameters on lifetime of the sensor node are sampling and transmission frequency. The lifetime of wireless Sensor node can be increased by decreasing these parameters.

Fig. 3 shows the effect of time and our model parameters (sampling frequency, transmission frequency, and processing power and transmission power) on the lifetime of a sensor 
node. In Fig. 3(a) effect of time, sampling frequency and transmission frequency is shown. Sampling frequency has a very low effect on the energy consumption of sensor node as compared to a transmission frequency that is why remaining lifetime of sampling frequency parameter is more than transmission frequency. In Fig. 3(a) we can see that the remaining lifetime of sensor during transmission frequency effect is near 2000 hours. However, during sampling frequency effect, the sensor node remaining lifetime is nearly 5800 hours. In Fig. 3(b), 3(c) effect of transmission power and effect of processing power is shown. Processing power has less effect on energy consumption as compared to transmission power that is why the remaining lifetime of processing power parameter is more than the transmission power parameter. In Fig. 3(b) we can see that the remaining lifetime of the sensor is 10000 during transmission power varying. However, during processing power effect, the sensor node remaining lifetime is nearly 10500 hours as shown in Fig. 3(c). Here the graphical representation of Fig. 3(b) and 3(c) are not very accurate because of scaling issues.

From all parameters, we can see the more effective parameters on wireless node energy consumption are sampling frequency and transmission frequency. So, wireless sensor energy consumption can be minimized by reducing both parameters which are highly affecting the battery of sensor node.

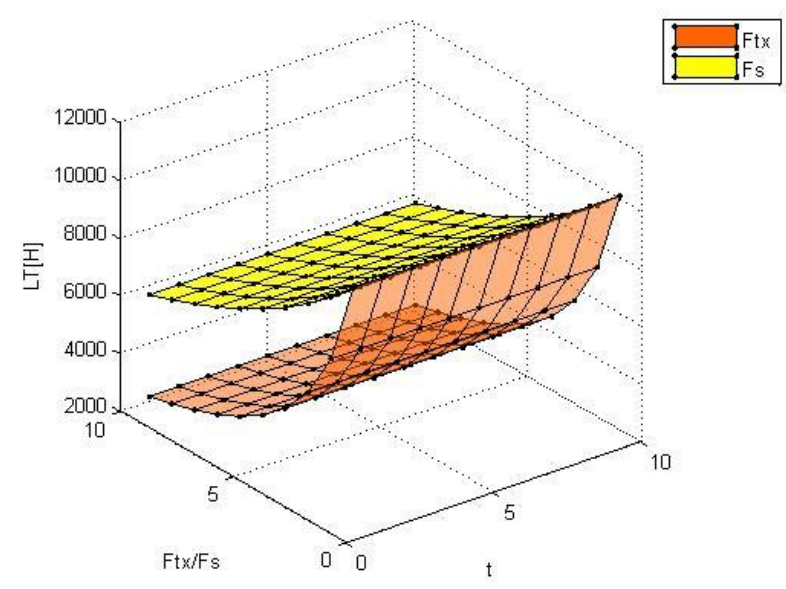

(a)

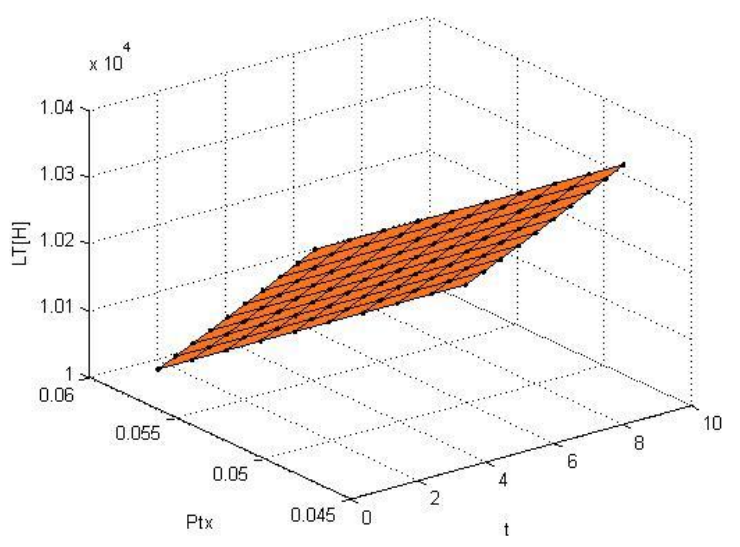

(b)

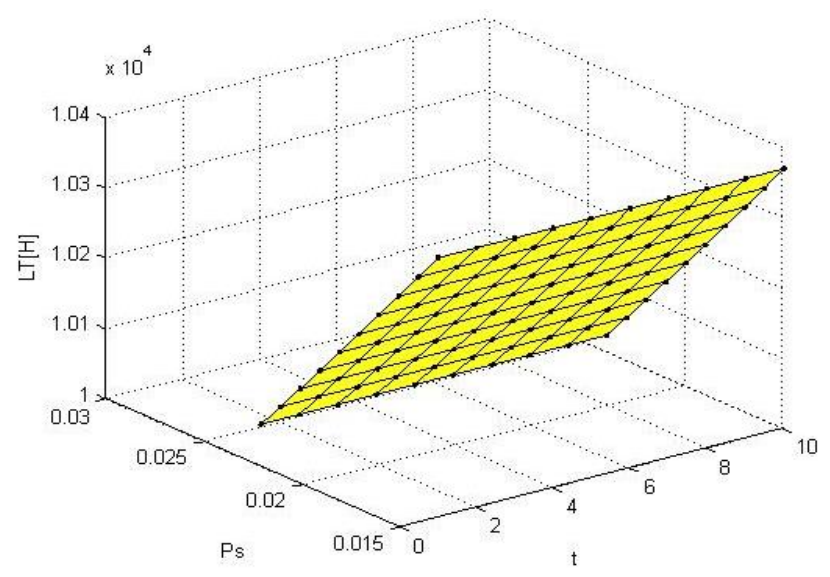

(c)

Fig. 3. Effect of time, frequencies and powers on node lifetime (a) Effect of time, sampling and transmission frequency on lifetime (b) Effect of time and transmission power on lifetime (c) Effect of time and processing power on lifetime.

Sensor lifetime totally depends on its energy consumption. The relation of sensor lifetime with energy consumption is given below:

Life time of sensor $\propto \frac{1}{\text { Total energy consumption of sensor }}$

According to the sensor lifetime relation, lifetime graphs are approximately resampled with the relation.

The overall goal is to derive a formulation for total energy consumption of wireless sensor node in order to get the best selection of wireless sensor parameters, which can reduce the energy consumption and enhance the battery life. As explained in graphical detail, we compared the impact of mainly four parameters on wireless sensor node which are the sampling frequency, transmission frequency, processing power and transmission power. According to Fig. 2 and 3, transmission frequency and sampling frequency have a less remaining lifetime as compared to processing power and transmission power. As per our model, WSN lifetime can be enhanced by reducing the transmission and sampling frequency parameters.

\section{CONCLUSION}

In this paper, we proposed a new and realistic energy model for WSN. Energy consumption of four WSN parameters (sampling frequency, transmission frequency, processing power and transmission power) are investigated. We analyzed the effects of these parameters on a lifetime of wireless sensor nodes. The results show that two parameters, sampling frequency, and transmission frequency are much more effective on energy consumption and lifetime of wireless sensor nodes as compared to processing and transmission power. By reducing sampling and transmission frequency, energy consumption of wireless sensor nodes can be reduced. We expect that our work will help to improve the lifetime performance of wireless sensor networks. 


\section{ACKNOWLEDGMENT}

This work is supported by the Higher Education Commission of Pakistan.

\section{REFERENCES}

[1] Gannapathy, Vigneswara Rao, MohdRiduan Ahmad, MohdKadimSuaidi, Muhammad Syahrir Johal, and ErykDutkiewicz. "Concurrent MAC with short signaling for multi-hop wireless mesh networks." In Ultra Modern Telecommunications \& Workshops, 2009. ICUMT'09. International Conference on, pp. 1-7. IEEE, (2009).

[2] Ibrahim, Tuani, Ahamed Fayeez, Vigneswara Rao Gannapathy, Md Isa, Ida Syafiza, Sarban Singh, Ranjit Singh, and Siva Kumar Subramaniam. "Throughput Analysis of Energy Aware Routing Protocol for Real-Time Load Distribution in Wireless Sensor Network (WSN)." Internaltional Journal of Reseach Engineering and Technology 2 (2013): 540-544.

[3] Wang, Andrew Y., and Charles G. Sodini. "A simple energy model for wireless microsensor transceivers." In Global Telecommunications Conference, 2004. GLOBECOM'04. IEEE, vol. 5, pp. 3205-3209. IEEE, (2004).

[4] Sha, Kewei, and Weisong Shi. "Modeling the lifetime of wireless sensor networks." Sensor Letters 3, no. 2 (2005): 126-135.

[5] Wang, Andrew Y., and Charles G. Sodini. "On the energy efficiency of wireless transceivers." In Communications, 2006. ICC'06. IEEE International Conference on, vol. 8, pp. 3783-3788. IEEE, (2006).

[6] Zheng, Meng, Haibin Yu, Jianying Zheng, and Peng Zeng. "Tradeoff between utility and lifetime in energy-constrained wireless sensor networks." Journal of Control Theory and Applications 8, no. 1 (2010): 75-80.

[7] Liu, Tao, Qingrui Li, and Ping Liang. "An energy-balancing clustering approach for gradient-based routing in wireless sensor networks."
Computer Communications 35, no. 17 (2012): 2150-2161.

[8] Kamyabpour, Najmeh, and Doan B. Hoang. "Modeling overall energy consumption in Wireless Sensor Networks." arXiv preprint arXiv:1112.5800 (2011).

[9] Kaurav, Jyoti, and Kaushik Ghosh. "Effect of Transmitting Radius, Coverage Area and Node Density on the Lifetime of a Wireless Sensor Network." (2012).

[10] Fayeez, A. T. I., V. R. Gannapathy, Ida S. Md Isa, M. K. Nor, and N. L. Azyze. "Literature review of battery-powered and solar-powered wireless sensor node." Asian Research Publishing Network-Journal of Engineering and Applied Sciences 10, no. 2 (2015): 671-677.

[11] AkanshaTomar and Prof.GauravBardwaj, "A survey on dynamic power management techniques in wireless sensor networks", INT.J.AREEIE, (2016), vol.5(6):2320-3765.

[12] Krishan Kumar Ms. Shruti Goel,"Literature Review to Optimize the Energy Consumption in Wireless Sensor Network" International Journal of Computer Science and Mobile Computing, Vol.6 Issue.7, July- (2017), pg. 106-113

[13] Rodrigues, Leonardo M., Carlos Montez, Gerson Budke, Francisco Vasques, and Paulo Portugal. "Estimating the Lifetime of Wireless Sensor Network Nodes through the Use of Embedded Analytical Battery Models." Journal of Sensor and Actuator Networks 6, no. 2 (2017): 8.

[14] Corporaton, Moteiv. "Tmote Sky: Datasheet." (2006).

[15] Amiri, Moslem. "Measurements of energy consumption and execution time of different operations on Tmote Sky sensor nodes." PhD diss., Masarykovauniverzita, Fakultainformatiky, (2010).

[16] DEVADIGA, Ketan. IEEE 802.15. 4 and the Internet of things.

[17] Sivagami, A., K. Pavai, D. Sridharan, and SAV Satya Murty. "Estimating the energy consumption of wireless sensor node: Iris." International J. of Recent Trends in Engineering and Technology 3, no. 4 (2010). 